Los Angeles, California, United States, 2. UCLA Luskin School of Public Affairs, Department of Social Welfare, Los Angeles, CA, USA, 3. UCSF School of Nursing, Institute for Health \& Aging; San Francisco, CA, USA, 4. UCSF School of Medicine, Division of Geriatrics; San Francisco, CA, USA

Purpose: At the 2017 IAGG World Congress, GSA presented an "innovative program," the AgeStage. Its aim was to contribute to the audience re-framing of aging to include creativity. Process: The Humanities and Arts Workgroup programmed intergenerational, live performances (30) as a visible venue attended by approximately 2,000 persons from 18 disciplines and 23 countries. Evaluation: Both online (438) and onsite (398) surveys were completed. Attendee's (91\%) rated the value of the experience as excellent or significant. The AgeStage "impact" was compiled through attendees' stated applications: curriculum and program development; advocacy; and options for their own aging. The audience highly rated the value of AgeStage performances to increased appreciation of older adults' creativity and to the importance of older persons' presence at scientific conferences. Qualitative responses enhanced understanding the experience: "The AgeStage engages the emotions and aspects of aging that can only be communicated holistically with art."

\section{WHAT'S THE STORY? QUALITATIVE RESPONSES TO THE AGESTAGE}

W. Lustbader, University of Washington School of Social Work, Burton, Washington, United States

We tend to remember and feel transformed by edifying experiences that tie into our emotions as well as our intellect. Among the over 200 write-in comments on the AgeStage evaluation forms, many of the attendees expressed their gratitude for the AgeStage experience as a stimulating change of emphasis. One comment exemplified this response: "Very powerful presentations! Some of the best in the conference." Many described being "moved" and often "inspired" by seeing, hearing and feeling older adults' creative expression through the arts. Several also provided descriptions about the way in which the AgeStage event reframed and/or enhanced their perceptions of aging: "Beyond \{the\} clinical-- this is the human and hopeful side to look at aging." Video clips from the performances will show how AgeStage attendees' experiential learning moved them to a fuller appreciation of older persons aging creatively.

\section{SESSION 2585 (SYMPOSIUM)}

PATTERNS OF SEDENTARY BEHAVIOR AND HEALTH OUTCOMES IN AGING WOMEN: THE OPACH STUDY Chair: A.Z. LaCroix, University of California, San Diego, La Jolla, California

Daily time spent sedentary is high among older adults, virtually impossible to accurately quantify using questionnaires, and accumulates in various patterns defined by how often breaks in sitting occur. No prospective studies have yet examined whether prolonged vs. short sedentary accumulation patterns increase risk for falls, incident diabetes or mortality in older women. The Objective Physical Activity and Cardiovascular Health (OPACH) Study enrolled 7048 ethnically-diverse postmenopausal women (average age 79; $33.5 \%$ Black, $17.6 \%$ Hispanic) from the Women's Health Initiative to measure physical activity and sedentary behavior using waist worn Actigraph GT3X accelerometers worn for up to 7 days; nearly 1,000,000 hours of activity data were collected among 6489 women who returned devices. Falls surveillance using monthly calendars collected for 1-year after accelerometer wear was conducted for 6580 women during which 5980 falls were reported among 2519 women. During up to 4.9 years of annual follow-up for disease endpoints and death, 280 incident cases of diabetes (physician diagnosed and treated with medication) and 499 deaths (162 CVD deaths) were ascertained. For each endpoint, we examined rate ratios (incident rate ratios or hazard ratios) adjusting for accelerometer wear-time (where appropriate), age, race/ethnicity, education, self-rated health, multimorbidity, physical functioning, alcohol consumption, smoking and accelerometer measured moderate-vigorous physical activity (MVPA). We also tested for interactions by MVPA and physical function. Taken together, results show the burden of morbidity and mortality associated with prolonged sitting and emphasize the need for public health interventions to interrupt sitting as part of aging well.

\section{SEDENTARY BEHAVIOR AND MORTALITY IN OLDER WOMEN}

A.Z. LaCroix ${ }^{1}$, J. Bellettiere, $\mathrm{PhD}^{2}$, M.J. LaMonte, $\mathrm{PhD}^{3}$, D.E. Rosenberg, $\mathrm{PhD}^{4}, 1$. University of California, San Diego, La Jolla, California, United States, 2. University of California, San Diego, La Jolla, California, USA; Center for Behavioral Epidemiology and Community Health, San Diego, California, USA, 3. University at Buffalo-SUNY, Buffalo, NY, USA, 4. Kaiser Permanente Washington Health Research Institute, Kaiser Permanente Washington, Seattle, Washington, USA

Results of associations between accelerometer-measured sedentary time and all-cause mortality are mixed and little is known about sedentary patterns and mortality. Accelerometers were used to measure sedentary time and mean sedentary bout duration for 4-to-7 days among 6113 women followed up to 4.9 years for all-cause and cardiovascular disease (CVD)-related mortality. We observed 499 deaths, 162 were CVD-related. Adjusting for age, race/ethnicity, education, self-rated health, multimorbidity, physical functioning, and alcohol and cigarette use, women in sedentary time quartiles (Q)2, Q3, Q4 (vs. Q1) had all-cause mortality hazard ratios (HR; 95\% confidence interval) of 1.24 (0.89-1.75), 1.35 (0.97-1.87), 1.85 (1.34-2.55); p-trend<.001. HRs for mean bout duration Q2, Q3, Q4 (vs. Q1) were 1.16 (0.83-1.62), 1.39 (1.02-1.89), 1.67 (1.232.26); p-trend<.001. All associations persisted after adjustment for moderate-to-vigorous physical activity and were notably stronger for CVD-mortality. Both total volume and patterns of sedentary time are related to mortality risk in older women.

\section{SEDENTARY BEHAVIOR: IMPORTANT TO HEALTH, DIFFICULT TO MEASURE}

M.J. LaMonte' ${ }^{1}$ E. Rillamas-Sun, $\mathrm{PhD}^{2}$, J. Bellettiere, $\mathrm{PhD}^{3}$, D.E. Rosenberg, $\mathrm{PhD}^{4}$, A.Z. LaCroix, $\mathrm{PhD}^{5}$, 1. Department 